\title{
Diversity of gamma-ray burst energetics vs. supernova homogeneity: SN 2013cq associated with GRB $130427 A^{\star, \star \star}$
}

A. Melandri ${ }^{1}$, E. Pian²,3,4, V. D’Elia ${ }^{5,6}$, P. D’Avanzo ${ }^{1}$, M. Della Valle ${ }^{7,8}$, P. A. Mazzali ${ }^{9,10,11}$, G. Tagliaferri ${ }^{1}$, Z. Cano $^{12}$, A. J. Levan ${ }^{13}$, P. Møller ${ }^{14}$, L. Amati ${ }^{3}$, M. G. Bernardini ${ }^{1}$, D. Bersier ${ }^{9}$, F. Bufano ${ }^{15}$, S. Campana ${ }^{1}$, A. J. Castro-Tirado ${ }^{16}$, S. Covino ${ }^{1}$, G. Ghirlanda ${ }^{1}$, K. Hurley ${ }^{17}$, D. Malesani ${ }^{18}$, N. Masetti ${ }^{3}$, E. Palazzi ${ }^{3}$, S. Piranomonte ${ }^{6}$, A. Rossi ${ }^{19}$, R. Salvaterra ${ }^{20}$, R. L. C. Starling ${ }^{21}$, M. Tanaka ${ }^{22}$, N. R. Tanvir ${ }^{21}$, and S. D. Vergani ${ }^{23}$

1 INAF - Osservatorio Astronomico di Brera, via E. Bianchi 46, 23807 Merate, Italy e-mail: andrea.melandri@brera.inaf.it

2 Scuola Normale Superiore, Piazza dei Cavalieri 7, 56126 Pisa, Italy

3 INAF - Istituto di Astrofisica Spaziale e Fisica Cosmica, via P. Gobetti 101, 40129 Bologna, Italy

${ }^{4}$ INFN, Sezione di Pisa, Largo Pontecorvo 3, 56127 Pisa, Italy

5 ASI, Science Data Centre, via del Politecnico snc, 00133 Roma, Italy

6 INAF - Osservatorio Astronomico di Roma, via Frascati 33, 00040 Monte Porzio Catone, Italy

7 INAF - Osservatorio Astronomico di Capodimonte, Salita Moiariello 16, 80131 Napoli, Italy

${ }^{8}$ ICRANET, Piazza della Repubblica 10, 65122 Pescara, Italy

9 ARI, Liverpool John Moores University, IC2 Liverpool Science Park 146 Brownlow Hill, Liverpool L3 5RF, UK

10 INAF - Osservatorio Astronomico di Padova, Vicolo dell' Osservatorio 5, 35122 Padova, Italy

11 Max-Planck Institute for Astrophysics, Garching, Karl-Schwarzschild-Str. 1, Postfach 1317, 85741 Garching, Germany

12 Centre of Astrophysics and Cosmology, Science Institute, University of Iceland, Dunhagi 5, 107 Reykjavik, Iceland

13 Department of Physics, University of Warwick, Coventry CV4 7AL, UK

14 European Southern Observatory, Karl-Schwarzschildstrasse 2, 85748 Garching bei München, Germany

15 Departamento de Ciencias Fisicas, Universidad Andres Bello, Avda. Republica 252, 837025 Santiago, Chile

16 Instituto de Astrofísica de Andalucía, Glorieta de la Astronomía s/n, 18008 Granada, Spain

17 University of California, Berkeley, Space Sciences Laboratory, 7 Gauss Way, Berkeley CA 94720-7450, USA

18 Dark Cosmology Centre, Niels Bohr Institute, University of Copenhagen, Juliane Maries Vej 30, 2100 Copenhagen, Denmark

19 Thüringer Landessternwarte Tautenburg, Sternwarte 5, 07778 Tautenburg, Germany

${ }^{20}$ INAF - Istituto di Astrofisica Spaziale e Fisica Cosmica Milano, via E. Bassini 15, 20133 Milano, Italy

21 Department of Physics and Astronomy, University of Leicester, University Road, Leicester LE1 7RH, UK

22 National Astronomical Observatory of Japan, Mitaka, 181-8588 Tokyo, Japan

23 GEPI, Observatoire de Paris, CNRS, Univ. Paris Diderot, 5 place Jules Janssen, 92195 Meudon, France

Received 4 February 2014 / Accepted 23 April 2014

\section{ABSTRACT}

\begin{abstract}
Aims. Long-duration gamma-ray bursts (GRBs) have been found to be associated with broad-lined type-Ic supernovae ( $\mathrm{SNe}$ ), but only a handful of cases have been studied in detail. Prompted by the discovery of the exceptionally bright, nearby GRB 130427A (redshift $z=0.3399$ ), we aim at characterising the properties of its associated SN2013cq. This is the first opportunity to test the progenitors of high-luminosity GRBs directly.

Methods. We monitored the field of the Swift long-duration GRB 130427A using the $3.6 \mathrm{~m}$ TNG and the $8.2 \mathrm{~m}$ VLT during the time interval between 3.6 and 51.6 days after the burst. Photometric and spectroscopic observations revealed the presence of the type Ic SN $2013 \mathrm{cq}$.

Results. Spectroscopic analysis suggests that SN 2013cq resembles two previous GRB-SNe, SN 1998bw and SN 2010bh, associated with GRB 980425 and X-ray flash (XRF) 100316D, respectively. The bolometric light curve of SN 2013cq, which is significantly affected by the host galaxy contribution, is systematically more luminous than that of SN 2010bh ( 2 mag at peak), but is consistent with SN 1998bw. The comparison with the light curve model of another GRB-connected SN 2003dh indicates that SN 2013cq is consistent with the model when brightened by $20 \%$. This suggests a synthesised radioactive ${ }^{56} \mathrm{Ni}$ mass of $\sim 0.4 M_{\odot}$. GRB $130427 \mathrm{~A} / \mathrm{SN} 2013 \mathrm{cq}$ is the first case of low- $z$ GRB-SN connection where the GRB energetics are extreme $\left(E_{\gamma \text {,iso }} \sim 10^{54} \mathrm{erg}\right)$. We show that the maximum luminosities attained by SNe associated with GRBs span a very narrow range, but those associated with XRFs are significantly less luminous. On the other hand the isotropic energies of the accompanying GRBs span 6 orders of magnitude $\left(10^{48}\right.$ erg $<E_{\gamma \text {,iso }}<$ $10^{54} \mathrm{erg}$ ), although this range is reduced when corrected for jet collimation. The GRB total radiated energy is in fact a small fraction of the SN energy budget.
\end{abstract}

Key words. gamma-ray burst: general - supernovae: individual: SN2013cq

* Based on observations made with the VLT, operated on the mountain of Cerro Paranal in Chile under programme 091.D-0291(A) and with the TNG, operated on the island of La Palma by the Fundación Galileo Galilei of the Instituto Nazionale di Astrofisica (INAF) at the Spanish Observatorio del Roque de los Muchachos of the Instituto de Astrofísica de Canarias under programme A27TAC_5.

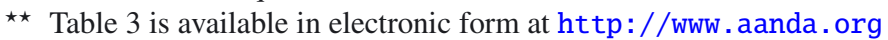




\section{Introduction}

The majority of nearby under-energetic long duration gammaray bursts and X-ray flashes (GRBs and $\mathrm{XRFs}^{1}$ with total isotropic energy $E_{\text {iso }}<10^{51} \mathrm{erg}$ ) are associated with with highly energetic supernovae (SNe; Mazzali et al. 2006a,b). The study of the $\mathrm{SN}$ in these cases is facilitated by the weakness of the GRB afterglow and the relatively small distance.

For $z \lesssim 0.3$, the accuracy of optical photometric and spectroscopic monitoring is satisfactory, and individual atomic species can be identified in the SN spectrum (Galama et al. 1998; Patat et al. 2001; Hjorth et al. 2003; Stanek et al. 2003; Soderberg et al. 2004; Malesani et al. 2004; Ferrero et al. 2006; Pian et al. 2006; Sollerman et al. 2006; Mirabal et al. 2006; Bersier et al. 2006; Cano et al. 2011a; Bufano et al. 2012; Melandri et al. 2012; Schulze et al. 2014). At higher redshifts, up to $z \sim 1$, the presence of an associated SN is inferred from the detection of a "rebrightening" in the late afterglow light curve due to the emerging SN component (Bloom et al. 1999; Castro-Tirado \& Gorosabel 1999; Galama et al. 2000; Castro-Tirado et al. 2001; Lazzati et al. 2001; Masetti et al. 2003; Zeh et al. 2004; Gorosabel et al. 2005; Bersier et al. 2006; Cobb et al. 2006; Soderberg et al. 2006; Tanvir et al. 2010; Cano et al. 2011b). In a few cases, single-epoch spectra obtained close to the peak of this rebrightening show SN features similar to those exhibited by GRB-accompanying SNe at lower redshifts (Della Valle et al. 2003; Garnavich et al. 2003; Greiner et al. 2004; Soderberg et al. 2005; Berger et al. 2011; Della Valle et al. 2006a; Sparre et al. 2011; Jin et al. 2013), then confirming the association of the GRB with a SN event.

All GRBs associated with $\mathrm{SNe}$ at $z \lesssim 0.3$ so far discovered show low isotropic energy, typically less than $\sim 10^{50} \mathrm{erg}$, with GRB 030329/SN 2003dh (Hjorth et al. 2003; Stanek et al. 2003; Matheson et al. 2003) being the only exception. This is a relatively nearby GRB $(z=0.168)$ with an isotropic energy of $E_{\gamma, \text { iso }} \sim 2 \times 10^{52} \mathrm{erg}$ (Vanderspek et al. 2004), which falls in the faint tail of the "cosmological" GRBs energy distribution (e.g. Amati et al. 2002; Amati et al. 2008). The long nearby GRB 130427A is exceptional and outstanding. It showed a huge isotropic energy $\left(E_{\text {iso }} \sim 10^{54} \mathrm{erg}\right.$; Maselli et al. 2014; Amati et al. 2013a), and at the same time the association with a SN was clear (SN 2013cq; de Ugarte Postigo et al. 2013). GRB 130427A follows the well known Amati $\left(E_{\text {peak }}-E_{\text {iso }}\right.$, Amati et al. 2002) and Yonetoku ( $E_{\text {peak }}-L_{\text {iso }}$, Yonetoku et al. 2004) correlations (Maselli et al. 2014). This made the study of the properties and evolution of SN 2013cq particularly interesting, since no very energetic GRB has ever been detected at relatively low redshift, so that this is the first occurrence of a connection between a $\mathrm{SN}$ and a GRB that has all the characteristics of a cosmological event.

In this paper we present the results of our photometric and spectroscopic campaign, covering $\sim 1.5$ months, carried out with the VLT and the TNG. Throughout the paper we assume a standard cosmology with $H_{0}=72 \mathrm{~km} \mathrm{~s}^{-1} \mathrm{Mpc}^{-1}, \Omega_{\mathrm{m}}=0.27$, and $\Omega_{\Lambda}=0.73$.

\section{GRB 130427A / SN 2013cq}

GRB 130427A was a long and extremely bright GRB $\left(T_{90} \sim\right.$ $160 \mathrm{~s}$; Barthelmy et al. 2013) that independently triggered the

\footnotetext{
1 XRFs are a softer version of GRBs, with integrated spectra peaking around 5-10 keV instead of 100-1000 keV.
}

Fermi satellite at $07^{\mathrm{h}} 47^{\mathrm{m}} 06^{\mathrm{S}} .42$ UT (von Kienlin 2013; Zhu et al. 2013) and the Swift satellite at $07^{\mathrm{h}} 47^{\mathrm{m}} 57^{\mathrm{s}} .5$ UT (Maselli et al. 2013), and attained the highest fluences observed in the $\gamma$-ray band for both satellites $\left(f_{\text {Swift }}(15-150 \mathrm{keV}) \sim 5 \times 10^{-4} \mathrm{erg} \mathrm{cm}^{-2}\right.$ and $\left.f_{\text {Fermi }}(0.01-20 \mathrm{MeV}) \sim 4 \times 10^{-3} \mathrm{erg} \mathrm{cm}^{-2}\right)$. The high-energy emission of GRB 130427A was also detected by several orbiting observatories, i.e. MAXI (Kawamuro et al. 2013), INTEGRAL (Pozanenko et al. 2013), Konus-Wind (Golenetskii et al. 2013), AGILE (Verrecchia et al. 2013), Suzaku (Akiyama et al. 2013), RHESSI (Smith et al. 2013), and Mars Odyssey ${ }^{2}$. This event has been the focus of several recently published studies (Ackermann et al. 2014; Preece et al. 2014; Maselli et al. 2014; Vestrand et al. 2014; Kouveliotou et al. 2013; Laskar et al. 2013; Perley et al. 2013; Panaitescu et al. 2013; Bernardini et al. 2014). The redshift was measured to be $z=0.3399 \pm 0.0002$ (Levan et al. 2013a; Xu et al. 2013a; Flores et al. 2013).

A bright flash in the optical band, probably due to a reverse shock component, was observed simultaneously with the high energy emission (>100 MeV) at very early times. Subsequently, the GRB afterglow emission can be described by the contribution of both reverse and forward shocks (Vestrand et al. 2014; Panaitescu et al. 2013; Maselli et al. 2014; Perley et al. 2013; Levan et al. 2013b).

GRB 130427A exploded in a relatively bright, extended host galaxy, catalogued in the Sloan Digital Sky Survey (SDSS J113232.84+274155.4), which showed the typical properties of the nearby GRB host population (Savaglio et al. 2009). Its stellar mass $\left(M_{*}=2.1 \pm 0.7 \times 10^{9} M_{\odot}\right)$ and mean population age ( $250 \mathrm{Myr})$ indicate a blue, young and low-mass galaxy (Perley et al. 2013). The afterglow of GRB 130427A is slightly offset from the centroid of its host galaxy $\left(\sim 0.83^{\prime \prime}\right.$, corresponding to $\sim 4 \mathrm{kpc}$ in projection at the redshift of the GRB) and apparently there is no strongly star-forming region underlying the GRB (Levan et al. 2013b).

Despite its relatively low redshift, which favoured the detection and follow-up of the associated SN 2013cq in the $R$ band (Xu et al. 2013b), GRB 130427A displayed all properties of more commonly observed high-redshift bursts. The extraordinarily high observed energetics of GRB 130427A and its closeness motivated our optical multi-band search and the intensive follow-up of its associated SN.

\section{Observations and data reduction}

We observed the field of SN 2013cq with the ESO $8.2 \mathrm{~m}$ Very Large Telescope (VLT) at Paranal Observatory equipped with FORS2 (imaging in the BVRI filters and spectroscopy) and with the Italian $3.6 \mathrm{~m}$ Telescopio Nazionale Galileo (TNG) equipped with DOLORES (imaging in the $g^{\prime} r^{\prime} i^{\prime}$ filters) from 3.6 to 51.6 days after the burst. Tables 1 and 3 summarise our observations.

\subsection{Imaging}

Image reduction, including de-biasing and flat-fielding, was carried out following standard procedures. Images were calibrated using a set of SDSS-catalogued stars acquired with SDSS $g^{\prime} r^{\prime} i^{\prime}$ filters (TNG observations) and with respect to standard fields in the BVRI filters (VLT observations). We performed differential photometry at the position of the optical afterglow, using an aperture of $\geq 2 \times F W H M$ of individual frames, which is large enough to include also the contribution of the underlying host galaxy. Observed $B V R I$ light curves are shown in Fig. 1.

\footnotetext{
2 http://www.ssl.berkeley.edu/ipn3/masterli.txt
} 


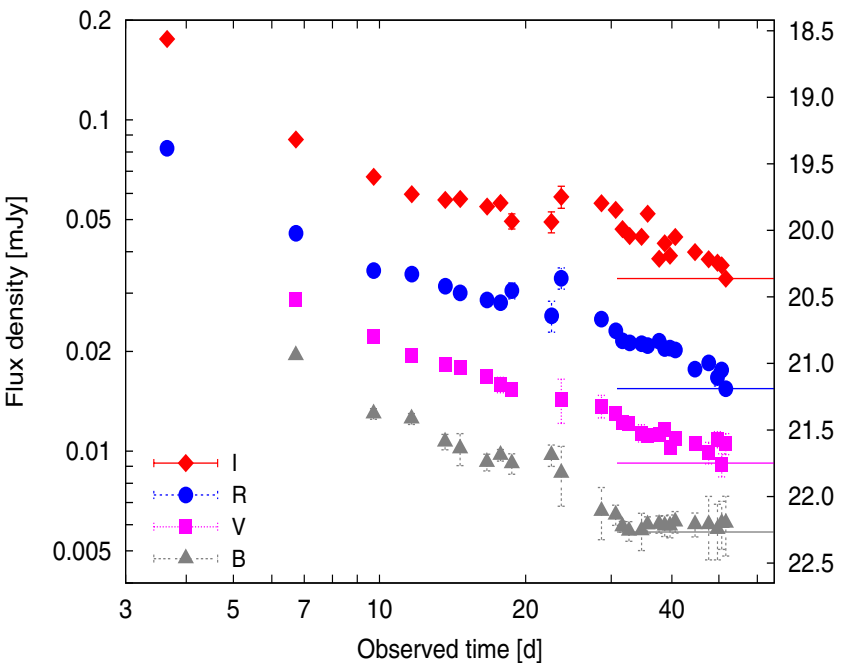

Fig. 1. Observed BVRI light curves of GRB 130427A/SN 2013cq, not corrected for Galactic extinction. For clarity, $R$ and $I$ magnitudes have been shifted by 0.45 and $0.75 \mathrm{mag}$, respectively. Horizontal lines represent the fluxes of the host galaxy in each filter.

During our campaign it was not possible to acquire a deep image of the host galaxy alone because the GRB optical counterpart dominates the emission at early epochs and may still contribute significantly at late epochs. Moreover, the offset of the $\mathrm{SN}$ with respect to the host galaxy is only $\sim 0.8^{\prime \prime}$ (Levan et al. $2013 \mathrm{~b}$ ), which is always comparable to the seeing of our images (see Table 3). Therefore, we used the SDSS magnitudes of the host galaxy that are $u=22.41 \pm 0.33, g=21.98 \pm 0.11$, $r=21.26 \pm 0.09, i=21.19 \pm 0.16$ and $z=21.11 \pm 0.54$. We converted these magnitudes into Johnson/Cousins magnitudes using the transformation equations in Jester et al. (2005), obtaining the final values $B_{\mathrm{HG}}=22.2, V_{\mathrm{HG}}=21.5, R_{\mathrm{HG}}=21.2$ and $I_{\mathrm{HG}}=20.5$. These values are consistent with Perley et al. (2013) and shown in Fig. 1.

We subtracted the estimated values for the host galaxy from our data, corrected for Galactic extinction (using the catalogued value of $E_{B-V, \text { Galactic }}=0.02 \mathrm{mag}$; Schlegel et al. 1998; Schlafly \& Finkbeiner 2011), applied k-corrections using our spectra (only $B$ and $V$ filters, because the spectra do not cover the redshifted $R$ and $I$ filters central wavelengths), and then subtracted the afterglow component. The temporal behaviour of the afterglow in each filter, where the early light curves were modelled with a forward relativistic shock into the circumstellar medium, can be described by a steepening power law with decay indices of $\sim 0.8$ and 1.5 before and after a break located at $\sim 0.5$ days (Laskar et al. 2013; Maselli et al. 2014; Perley et al. 2013; Xu et al. 2013b). Our data cover the phase after this temporal break and are consistent with the above time decay until a week after explosion, when the contribution from SN 2013cq becomes increasingly important. The afterglow model was then subtracted from the host-subtracted light curves and the residual, which is attributed to SN 2013cq alone, was corrected for intrinsic absorption following $\mathrm{Xu}$ et al. (2013b). These authors estimated the value $E_{(B-V) \text {, Host Galaxy }}=0.05 \mathrm{mag}$ from the detection of NaID 5890 \& 5896 absorption lines. The final corrected VRI light curves are reported in Fig. 2. The $B$-band light curve is heavily contaminated by the host galaxy and therefore not meaningful and not shown. The dereddened magnitudes have been transformed into monochromatic fluxes using the zeropoints in Fukugita et al. (1995).
Table 1. Journal of the VLT/FORS2 spectroscopic observations of SN 2013cq.

\begin{tabular}{lccccc}
\hline \hline Date & $\Delta t_{\mathrm{obs}}$ & $t_{\exp }$ & $\langle S / N\rangle$ & Grism & $\Delta t_{\mathrm{RF}}$ \\
\hline & (days) & $(\mathrm{s})$ & & & (days) \\
\hline 2013 May 4 & 6.737 & $1 \times 900$ & $13-28$ & $300 \mathrm{~V}$ & +5.02 \\
2013 May 4 & 6.756 & $1 \times 900$ & $2-6$ & $300 \mathrm{I}$ & +5.04 \\
2013 May 7 & 9.735 & $1 \times 900$ & $4-10$ & $300 \mathrm{~V}$ & +7.26 \\
2013 May 10 & 13.669 & $1 \times 900$ & $6-10$ & $300 \mathrm{I}$ & +10.20 \\
2013 May 12 & 15.660 & $2 \times 900$ & $5-12$ & $300 \mathrm{~V}$ & +11.68 \\
2013 May 14 & 16.680 & $2 \times 1800$ & $10-20$ & $300 \mathrm{~V}$ & +12.44 \\
2013 May 16 & 18.696 & $2 \times 900$ & $3-9$ & $300 \mathrm{~V}$ & +13.95 \\
2013 May 25 & 28.676 & $2 \times 900$ & $3-5$ & $300 \mathrm{~V}$ & +21.40 \\
2013 May 27 & 30.663 & $2 \times 1800$ & $7-13$ & $300 \mathrm{~V}$ & +22.88 \\
2013 May 28 & 31.665 & $2 \times 1800$ & $10-15$ & $300 \mathrm{~V}$ & +23.63 \\
\hline
\end{tabular}

Notes. $\Delta t_{\mathrm{obs}}$ : acquisition starting time of the spectra in the observer frame, with respect to the GRB trigger time; $t_{\text {exp }}$ : total exposure. The $\langle S / N\rangle$ is the average per spectral bin between the $\mathrm{S} / \mathrm{N}$ at the edges of the grism, where efficiency is lower, and that at the central wavelengths. The last column shows the rest-frame time.

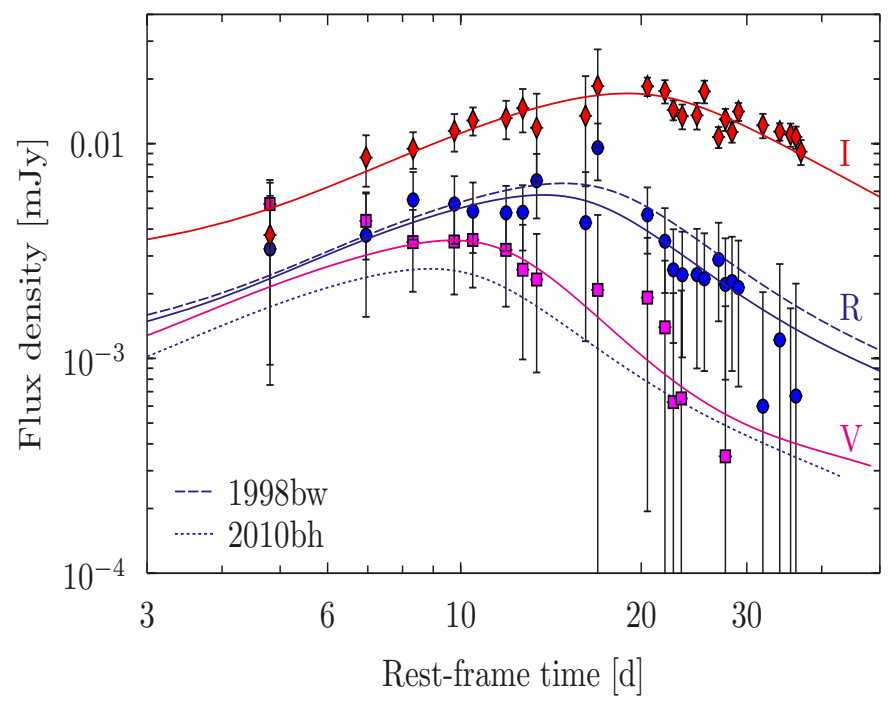

Fig. 2. SN 2013cq rest-frame light curve for the $V$ (magenta squares), $R$ (blue circles), and $I$ (red diamonds) bands. We also show the k-corrected $R$ band light curves of SN 1998bw (dashed line) and SN 2010bh (dotted line). Solid coloured lines are the $k$-corrected light curves of SN 1998bw transformed by stretch and luminosity factors and represent the best fit to the SN 2013cq VRI light curves.

\subsection{Spectroscopy}

VLT/FORS2 spectroscopy was carried out using the $300 \mathrm{~V}$ grism, covering the range 4450-9500 (corresponding to $3320-7090 \AA$ in the GRB rest-frame). For two epochs we also used the 300I grism, covering the range $6000-11000 \AA$ (4478-8210 rest-frame). In all cases we used a $1^{\prime \prime}$ slit, resulting in an effective resolution $R=440$ at the central wavelengths $\lambda_{300 \mathrm{~V}}=5900 \AA$ and $\lambda_{300 \mathrm{I}}=8600 \AA$, respectively. The spectra were extracted using standard procedures within the packages ESO-MIDAS ${ }^{3}$ and IRAF $^{4}$. A He-Ar lamp and spectrophotometric stars were used to calibrate the spectra in wavelength and flux, respectively. We accounted for slit losses by matching the flux-calibrated spectra to our simultaneous multi-band photometry.

\footnotetext{
3 http://www.eso.org/projects/esomidas/

4 http://iraf.noao.edu/
} 
After subtracting the contribution of the host galaxy using a spline interpolation to the SDSS magnitudes, we corrected the residual for Galactic extinction (with the extinction curve of Cardelli et al. 1989). We also applied the correction for the intrinsic reddening following Xu et al. (2013b) as in Sect. 3.1. We model the afterglow spectrum with a single power law as

$F_{\lambda}(\lambda)=N \times\left(\frac{\lambda_{\text {norm }}}{\lambda}\right)^{\beta_{\lambda}}$,

where we fixed $\lambda_{\text {norm }}$ to the rest-frame flux at $6588 \AA$ (corresponding to the $R$ band), $N$ is the power law normalisation and the spectral index $\beta_{\lambda}=1.5$ (Maselli et al. 2014; Perley et al. 2013). Telluric absorption features and the noisier parts of the spectra $\left(\lambda_{\mathrm{RF}}<3400 \AA\right.$ and $\lambda_{\mathrm{RF}}>6800 \AA$ for the $300 \mathrm{~V}$ grism and $\lambda_{\mathrm{RF}}<4600 \AA$ for the 300I grism) have been omitted. A clearly spurious feature has been deleted from the spectrum acquired at +11.68 rest-frame days.

\section{Results}

\subsection{Optical light curves}

Inspection of Fig. 1 shows a rebrightening at $\sim 20$ days, more pronounced in the redder filters, that is the tell-tale signature of an underlying SN, first identified by Xu et al. (2013a) and named SN 2013cq (de Ugarte Postigo et al. 2013). In Fig. 2 we show the final VRI light curves of SN $2013 \mathrm{cq}$.

Also plotted in Fig. 2 are the $k$-corrected, $R$ band light curves of SN 1998bw and SN 2010bh, which were associated with GRB 980425 (Galama et al. 1998) and XRF 100316D (Starling et al. 2011; Cano et al. 2011a; Bufano et al. 2012; Olivares et al. 2011), respectively, as they would appear if they occurred at a redshift of $z=0.3399$. To determine the rest-frame peak times in each filter, we adopted the formalism of Cano (2013), by which the optical light curves of the well observed type Ic SN 1998bw are used as templates to describe $\mathrm{SNe}$ with less well sampled light curves. The solid lines in Fig. 2 are the k-corrected light curves of SN 1998bw in the relevant bands, stretched in time and flux to match SN $2013 \mathrm{cq}$. The scaling factors were obtained with a best fit to the data of SN 2013cq, following Cano (2013). The peaks of these template light curves represent our best estimates of the light maxima of SN 2013cq: $T_{\text {peak }, V} \sim 9.6 \pm 0.7$, $T_{\text {peak }, R} \sim 13.8 \pm 0.9$, and $T_{\text {peak }, I} \sim 17.9 \pm 1.4$ days after the burst. We note that the errors are formal uncertainties returned by the fit and are likely to underestimate the real uncertainties by about a factor of 2 . Our best-fit $R$ band maximum flux and time agree with those determined by $\mathrm{Xu}$ et al. (2013b) within the error bars. The SN 2013cq flux maximum in the $R$ band is found to be slightly fainter ( 0.2 mag) than SN 1998bw.

It is commonly observed in $\mathrm{SNe}$ of all types, including nearby Ic SNe and GRB/XRF SNe (Richmond et al. 1996; Foley et al. 2003; Mazzali et al. 2002, 2007; Valenti et al. 2008; Galama et al. 1998; Soderberg et al. 2004, 2006; Bufano et al. 2012), that the light maximum is reached later in redder bands. In SN 2013cq, this temporal evolution is particularly fast, so that the rise in the $V$ band is rapid and resembles the one of XRF SNe (Pian et al. 2006; Ferrero et al. 2006; Mirabal et al. 2006; Bufano et al. 2012; Olivares et al. 2011), while in $R$ and $I$ bands the rise is more like to that of SNe associated with low- $z$ underluminous GRBs and classical GRBs at higher redshifts (Galama et al. 1998; Patat et al. 2001; Garnavich et al. 2003; Malesani et al. 2004; Della Valle et al. 2006b; Clocchiatti et al. 2011; Melandri et al. 2012).

\subsection{Optical spectra}

In Fig. 3 we show the FORS2 spectra acquired between $\sim 5.0$ and $\sim 23.6$ rest-frame days after the burst event. The quality of our spectra is not sufficient for accurately measuring of individual absorption features and for estimating photospheric velocities, but only for a general comparison with the spectra of high kinetic energy Type Ic SNe. Among these we have selected some with good $\mathrm{S} / \mathrm{N}$ and/or spectral coverage: the GRB SNe 1998bw (Patat et al. 2001) and 2003dh (Hjorth et al. 2003; Mazzali et al. 2002), XRF SNe 2006aj (Pian et al. 2006), 2010bh (Bufano et al. 2012), and the broad-lined Ic SN2010ah (Mazzali et al. 2013). A similarity is found with SN 2010bh (see also Xu et al. 2013b) and with SN 1998bw, and for clarity we only show the comparison with these two SNe in Fig. 3. The spectra of SN 2010bh are a good match to those of SN 2013cq, although those of SN 1998bw are a better representation in the blue, especially at phases $+12.44,+21.40$, and +22.88 days, suggesting strong line-blocking from high-velocity material.

\subsection{Bolometric light curve}

We have constructed a bolometric light curve in the range 3000-10000 A using the available photometry. For each epoch we followed the reduction procedure described in Sect. 3. Then we fitted with a spline function the residual monochromatic light curves, which represent the SN component, and integrated the broad-band flux at each photometric observation epoch. The flux was linearly extrapolated blueward of the $V$-band flux down to $3000 \AA$ and redward of the $I$-band flux to $10000 \AA$. The result is reported in Fig. 4. The errors associated with our photometry, galaxy measurement, afterglow fit and intrinsic absorption were propagated and summed in quadrature. These are extremely large at the earlier epochs, so that the points have been omitted in the figure. For comparison and as a consistency check, we report the bolometric point obtained from the HST measurements of May 20, 2013 (Levan et al. 2013b). We also compare the bolometric light curve of SN 2013cq with those of SN 1998bw (Patat et al. 2001), SN 2006aj (Pian et al. 2006) and with the models for SN 2003dh (Mazzali et al. 2003) and SN 2012bz (Melandri et al. 2012). The bolometric light curve of SN 1998bw was constructed in the same rest-frame band (3000-10000 $\AA$ ) and using a Galactic extinction of $E_{B-V}=0.052$ mag as recently reported by Schlafly \& Finkbeiner (2011). It does not differ significantly from the one reported in Pian et al. (2006), which was corrected for a lower estimate of Galactic extinction and included a $~ 15 \%$ flux correction for NIR contribution. The model of the light curve of SN 2003dh (Mazzali et al. 2003) was rescaled by $20 \%$ to match the HST point, which is very accurate. The rescaled model also fits the rest of the light curve well, within the large errors. This suggests that the ${ }^{56} \mathrm{Ni}$ mass synthesized by SN $2013 \mathrm{cq}$ is $\sim 20 \%$ higher than that of SN 2003dh, which leads to an estimate of $\sim 0.4 M_{\odot}$ (Mazzali et al. 2006a).

\subsection{GRBs-SNe properties and correlations}

GRB 130427A is the brightest GRB detected by Swift/BAT, one of the most energetic ever $\left(E_{\gamma \text {,iso }} \sim 10^{54} \mathrm{erg}, E_{\gamma \text {,peak }} \sim 1.2-1.3 \times\right.$ $\left.10^{3} \mathrm{keV}\right)$, and the most energetic $\mathrm{GeV}$ emitting GRB $(\sim 125 \mathrm{GeV}$ in rest frame; Ackermann et al. 2014). GRB 130427A is located in a still unexplored region of the $E_{\gamma \text {,iso }}-E_{\gamma \text {,peak }}$ plane for low $z$ GRBs associated with SNe, yet it follows the Amati and Yonetoku correlations very well (see Fig. S6 in Maselli et al. 2014). Prior to SN 2013cq only one nearby SN was associated 
A. Melandri et al.: Diversity of GRB energetics vs. SN homogeneity
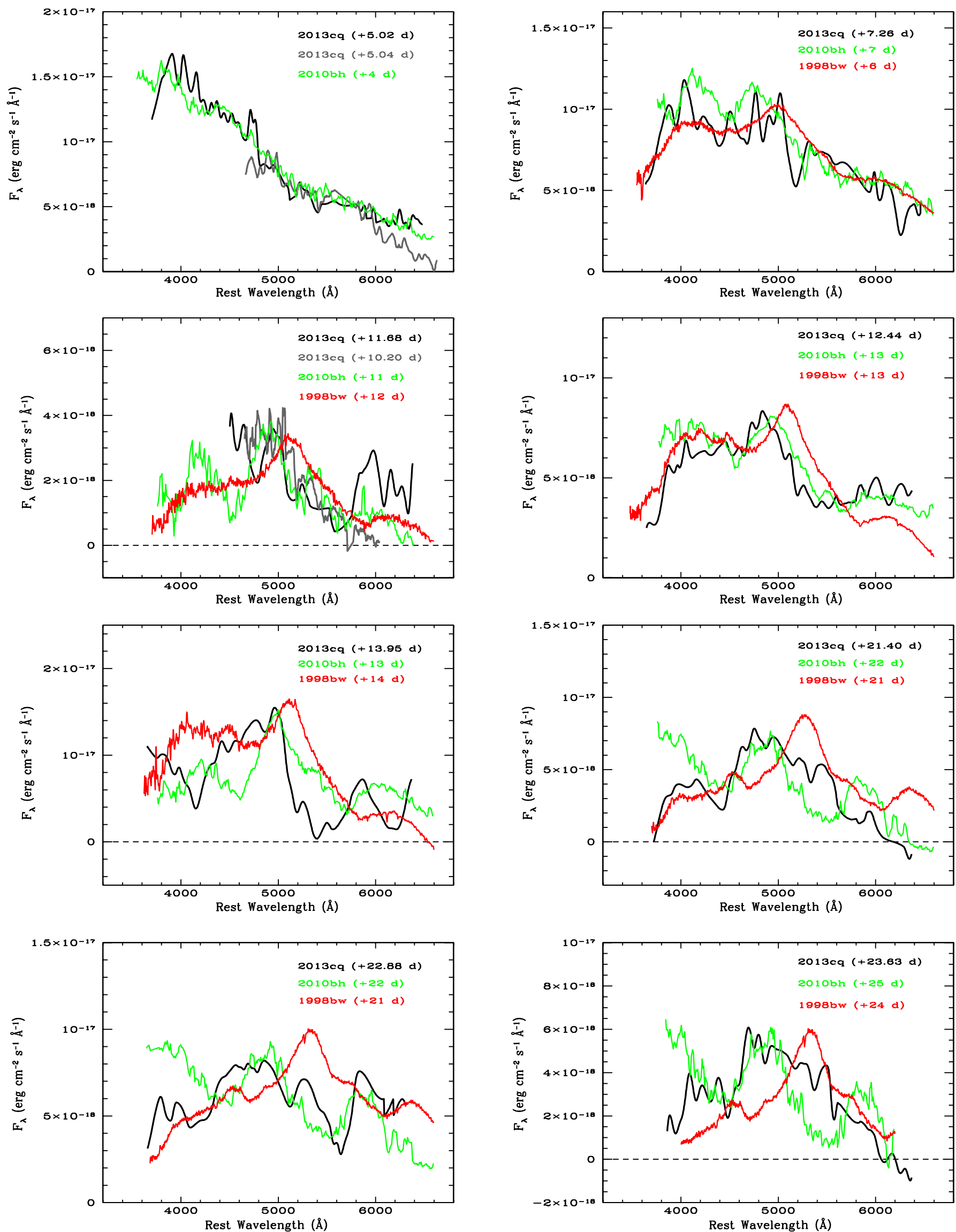

Fig. 3. Spectral evolution of SN 2013cq. Each panel shows the signal obtained with VLT-300V grism from SN 2013cq (black) and the comparison with SN2010bh (green) and SN 1998bw (red) at comparable rest frame phases. We superimpose, when available, the signal obtained with the VLT-300I grism (gray). All spectra have been smoothed with a boxcar of $15 \AA$. 
Table 2. GRBs-SNe properties.

\begin{tabular}{llclc}
\hline \hline GRB - SN & $z$ & $E_{\gamma, \text { iso }}$ & $M_{\text {bol }}$ & Refs. \\
\hline \multicolumn{5}{c}{$\left(\times 10^{52} \mathrm{erg}\right)$} \\
\hline $980425-1998 \mathrm{bw}$ & 0.0085 & $0.00010 \pm 0.00002$ & $-18.65 \pm 0.20$ & 1,2 \\
$030329-2003 \mathrm{dh}$ & 0.1687 & $1.5 \pm 0.3$ & $-18.71 \pm 0.15$ & 3,4 \\
$031203-2003 \mathrm{w}$ & 0.1055 & $0.010 \pm 0.004$ & $-18.92 \pm 0.20$ & 5,1 \\
$060218-2006 \mathrm{aj}$ & 0.0334 & $0.0053 \pm 0.0003$ & $-18.16 \pm 0.20$ & $4,6,7$ \\
$100316 \mathrm{D}-2010 \mathrm{bh}$ & 0.0591 & $0.007 \pm 0.003$ & $-17.50 \pm 0.25$ & 8,9 \\
$120422 \mathrm{~A}-2012 \mathrm{bz}$ & 0.283 & $0.024 \pm 0.008$ & $-18.56 \pm 0.15$ & 10 \\
$130427 \mathrm{~A}-2013 \mathrm{cq}$ & 0.3399 & $81.0 \pm 8.0$ & $-18.91 \pm 0.20$ & 11,2 \\
$130702 \mathrm{~A}-2013 \mathrm{dx}$ & 0.145 & $0.065 \pm 0.010$ & $-18.51 \pm 0.15$ & 12,13 \\
\hline
\end{tabular}

Notes. Columns: (1) GRB-SN name, (2) redshift, (3) isotropic $\gamma$-ray energy in the 1-10 $000 \mathrm{keV}$ energy band, and (4) peak bolometric magnitude of the SN.

References. 1) Amati et al. (2006); 2) this work; 3) Deng et al. (2005); 4) Amati et al. (2008); 5) Mazzali et al. (2006a); 6) Pian et al. (2006); 7) Ferrero et al. (2006); 8) Bufano et al. (2012); 9) Starling et al. (2011); 10) Melandri et al. (2012); 11) Maselli et al. 2014; 12) D'Elia et al. (in prep.); 13) Amati et al. (2013b; note that uncertainty on $E_{\gamma, \text { iso }}$ is amended here).

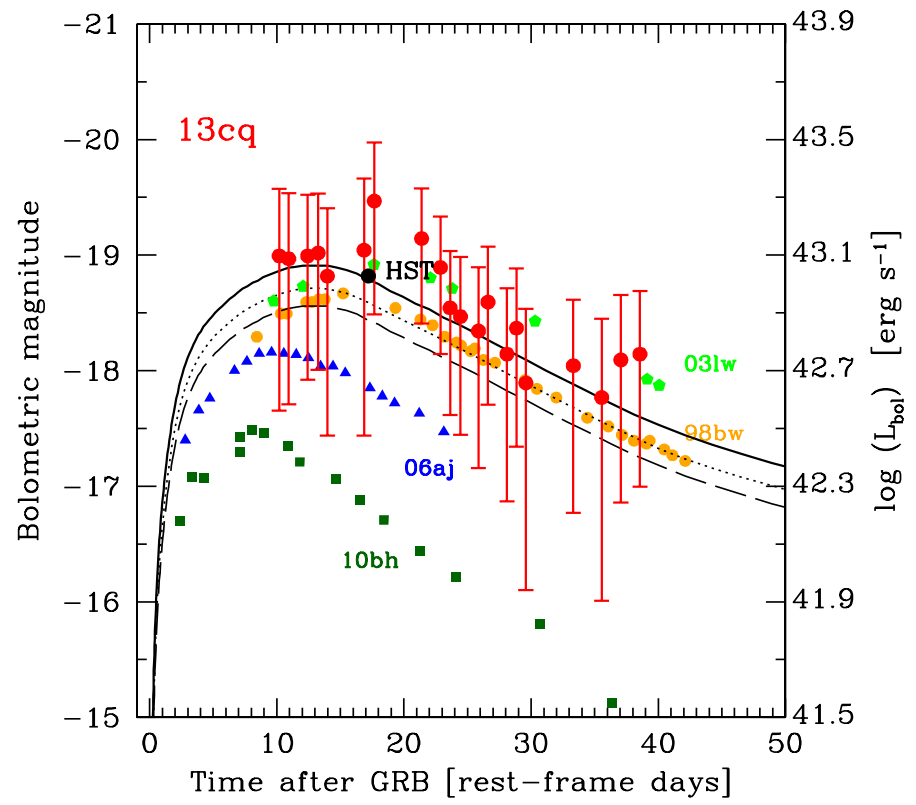

Fig. 4. Bolometric light curve of SN 2013cq (red filled circles) compared with those of SN 1998bw (orange filled circles), SN 2006aj (blue filled triangles), SN 2010bh (green filled squares), and with the models for SN 2003dh (dotted line) and SN 2012bz (dashed line). We also show the best fit model for SN 2013cq (solid line). As a consistency check, we report the bolometric point (black filled circle) obtained from the HST measurements of May 20, 2013. The error on this point is within the size of the symbol.

with a GRB having energetics similar to cosmological gammaray bursts, i.e. GRB $030329\left(E_{\gamma \text {,iso }} \sim 2 \times 10^{52} \mathrm{erg}, E_{\gamma \text {,peak }}=\right.$ $82 \pm 3 \mathrm{keV}$; Vanderspek et al. 2004). However, the properties of the GRB 030329 were less extreme than those observed for GRB 130427A.

A striking result when comparing GRB and SN properties (see Table 2) is that, while the values of $E_{\text {iso }}$ of the GRB span nearly six orders of magnitude ( $\sim 3$ after correcting for collimation effects) the SNe maximum luminosities $\left(M_{\mathrm{bol}}\right)$, which trace the mass of radioactive ${ }^{56} \mathrm{Ni}$ and correlate (like the $\mathrm{SN}$ kinetic energies) with the progenitor masses (Mazzali et al. 2013), are

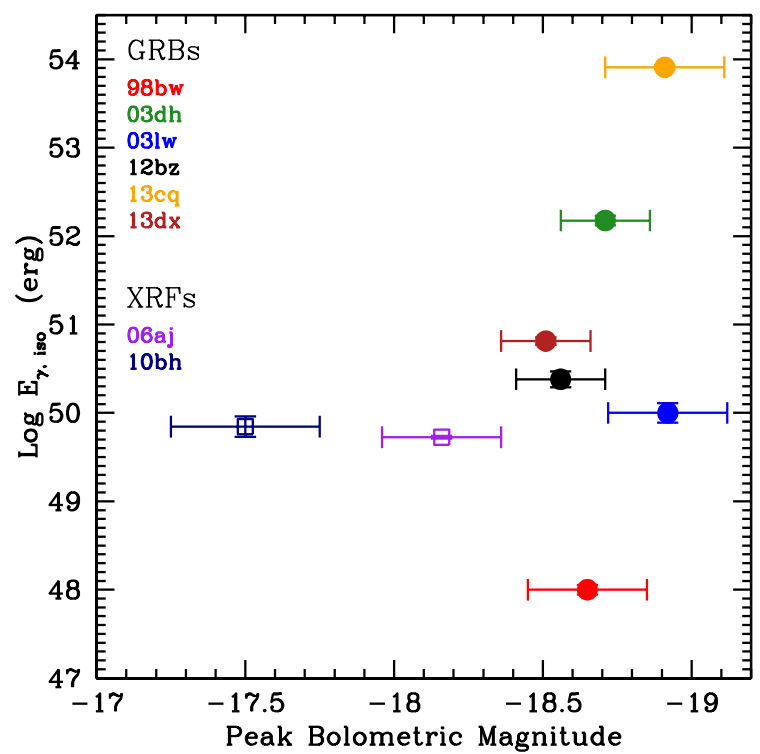

Fig. 5. GRB $E_{\gamma \text {,iso }}$ versus the peak magnitude of the bolometric light curve of the corresponding SN: the lack of correlation is apparent, where the SN brightness is nearly constant, while the GRB energy spans several orders of magnitude.

distributed in a narrow range $(\sim 0.5 \mathrm{mag})$ and can be considered roughly constant. On the other hand, nearby XRFs have total isotropic-equivalent energies similar to those of the less energetic GRBs, but their SNe have lower luminosities (Fig. 5).

\section{Conclusion}

The $R$ band light curve reported by Xu et al. (2013b, see also Perley et al. 2013) is $\sim 0.2$ mag fainter than that of SN 1998bw at maximum (Patat et al. 2001). Our analysis seems to confirm this (Fig. 2). However, the bolometric light curve (Fig. 4), and especially the accurate HST measurement (Levan et al. 2013b), suggest that SN 2013cq is marginally brighter than SN 1998bw and significantly more luminous ( 1.5 mag) than SN 2010bh. This points to the need to refer to bolometric rather than monochromatic information in the comparison of SN physical quantities.

Although the $\mathrm{S} / \mathrm{N}$ of the spectra is very limited, we note a some similarity with SN 2010bh (see also Xu et al. 2013b) and with SN 1998bw. In either case, this points to a classification of SN $2013 \mathrm{cq}$ as a broad line type Ic SN, as seen so far for all GRB/XRF SNe, and in turn to a massive, highly envelopestripped progenitor.

This is in line with the finding that $\mathrm{SNe}$ associated with GRBs - both underluminous and highly energetic - have all comparable luminosities and are more luminous than the two known low $z$, spectroscopically identified XRF/SNe 2006aj and 2010bh (Melandri et al. 2012). The extended host galaxy of GRB 130427A/SN2013cq is similar to the host galaxies of GRB 100316D/SN 2010bh and GRB 980425/SN 1998bw. The other ones known at low redshift are instead associated with smaller galaxies (Starling et al. 2011). However, there is no apparent correlation between the brightness of the GRB, the associated SNe, and their host galaxies (Levesque et al. 2010).

Owing to the limited S/N of the spectra of SN 2013cq, it is difficult to evaluate the photospheric velocity evolution of this $\mathrm{SN}$ and therefore its kinetic energy. Xu et al. (2013b) estimate $\sim 6 \times 10^{52} \mathrm{erg}$, i.e. similar to SN $1998 \mathrm{bw}$, which is plausible, considering the similarity of spectra and bolometric light curve. The data reported by Maselli et al. (2013b, their Fig. 2) show a break 
in the afterglow light curve, which they interpret as a jet break. If this is the case (jet collimation-correction can be complex, Campana et al. 2007; Perley et al. 2013), after correcting for the corresponding jet opening angle of about $3^{\circ}$, the total energy of GRB 130427 A decreases to $3 \times 10^{50} \mathrm{erg}$, which is less than $1 \%$ of the kinetic energy associated with its SN. A similar finding has been reported for other GRB-SN associations (Woosley \& Bloom 2006, Amati et al. 2007). Therefore it is very plausible that the SN may drive the GRB jet. The monitoring of nearby energetic GRBs is a critical test bed for confirming SN-driven GRB models or opening more exotic (and more energetic) scenarios based on black hole formation.

Acknowledgements. We thank the anonymous referee for valuable comments and suggestions that improved the paper. We thank the TNG staff, in particular G. Andreuzzi, L. Di Fabrizio, and M. Pedani, for their valuable support with TNG observations, and the Paranal Science Operations Team, in particular H. Boffin, S. Brillant, C. Cid, O. Gonzales, V. D. Ivanov, D. Jones, J. Pritchard, M. Rodrigues, L. Schmidtobreick, F. J. Selman, J. Smoker, and S. Vega. The Dark Cosmology Centre is funded by the Danish National Research Foundation. F.B. acknowledges support from FONDECYT through Postdoctoral grant 3120227 and from Project IC120009 "Millennium Institute of Astrophysics (MAS)" of the Iniciativa Científica Milenio del Ministerio de Economía, Fomento y Turismo de Chile. A.J.C.T. thanks the Spanish Ministry's Research Project AYA 201239727-C03-01. R.L.C.S. is supported by a Royal Society fellowship. D.M. acknowledges the Instrument Center for Danish Astrophysics (IDA) for support. This research was partially supported by contracts ASI INAF I/004/11/1, ASI INAF I/088/06/0, INAF PRIN 2011, and PRIN MIUR 2010/2011.

\section{References}

Ackermann, M., Ajello, M., Asano, K, et al. 2014, Science, 343, 42 Akiyama, M., Yamauchi, M., Ohmori, N., et al. 2013, GRB Coordinates Network, Circular Service, 14524

Amati, L. 2006, MNRAS, 372, 233

Amati, L., Frontera, F., Tavani, M., et al. 2002, A\&A, 390, 81

Amati, L., Della Valle, M., Frontera, F., et al. 2007, A\&A, 463, 913

Amati, L., Guidorzi, C, Frontera, F., et al. 2008, MNRAS, 391, 577

Amati, L., Dichiara, S., Frontera, F., \& Guidorzi, C. 2013a, GRB Coordinates Network, Circular Service, 14503

Amati, L., Dichiara, S., Frontera, F., et al. 2013b, GRB Coordinates Network, Circular Service, 15025

Barthelmy, S., Baumgartner, W. H., Cummings, J. R., et al. 2013, GRB Coordinates Network, Circular Service, 14470

Berger, E., Chornock, R., Holmes, T. R., et al. 2011, ApJ, 743, 204

Bernardini, M. G., Campana, S., Ghisellini, G., et al. 2014, MNRAS, 439, 80

Bersier, D., Fruchter, A. S., Strolger, L.-G., et al. 2006, ApJ, 643, 284

Bloom, J. S., Kulkarni, S. R., Djorgovski, S. G., et al. 1999, Nature, 401, 453

Bufano, F., Pian, E., Sollerman, J., et al. 2012, ApJ, 753, 67

Campana, S., Guidorzi, C., Tagliaferri, G., et al. 2007, A\&A, 472, 395

Cano, Z. 2013, MNRAS, 434, 1098

Cano, Z., Bersier, D., Guidorzi, C., et al. 2011a, ApJ, 740, 41

Cano, Z., Bersier, D., Guidorzi, C., et al. 2011b, MNRAS, 413, 669

Cardelli, J. A., Clayton, G. C., \& Mathis, J. 1989, ApJ 345, 245

Castro-Tirado, A. J., \& Gorosabel, J. 1999, A\&AS, 138, 449

Castro-Tirado, A. J., Sokolov, V. V., Gorosabel, J., et al. 2001, A\&A, 370, 398

Clocchiatti, A., Suntzeff, N. B., Covarrubias, R., \& Candia, P. 2011, AJ, 141, 163

Cobb, B. E., Bailyn, C. D., van Dokkum, P. G., \& Natarajan, P., 2006, ApJ, 645, 113

de Ugarte Postigo, A., Xu, D., Leloudas, G., et al. 2013, CBET, 3529, 1

Della Valle, M., Malesani, D., Benetti, S., et al. 2003, A\&A, 406, L33

Della Valle, M., Chincarini, G., Panagia, N., et al. 2006a, Nature, 444, 1050

Della Valle, M., Malesani, D., Bloom, J. S., et al. 2006b, ApJ, 642, 103

Deng, J, Tominaga, N., Mazzali, P. A., Maeda, K., \& Nomoto, K. 2005, ApJ, 624,898

Ferrero, P., Kann, D. A., Zeh, A., et al. 2006, A\&A, 457, 857

Flores, H., Covino, S., Xu, D., et al. 2013, GRB Coordinates Network, Circular Service, 14491

Foley, R. J., Papenkova, M. S., Swift, B. J., et al. 2003, PASP, 115, 1220

Fukugita, M., Shimasaku, K., \& Ichikawa, T. 1995, PASP, 107, 945
Galama, T. J., Vreeswijk, P. M., van Paradijs, J., et al. 1998, Nature, 395, 670

Galama, T. J., Tanvir, N., Vreeswijk, P. M., et al. 2000, ApJ, 536, 185

Garnavich, P. M., Stanek, K. Z., Wyrzykowski, L., et al. 2003, ApJ, 582, 924

Golenetskii, S., Aptekar, R., Frederiks, D., et al. 2013, GRB Coordinates

Network, Circular Service, 14487, 1

Gorosabel, J., Fynbo, J. P. U., Fruchter, A., et al. 2005, A\&A, 437, 411

Greiner, J., Klose, S., Salvato, M., et al. 2003, ApJ 599, 1223

Hjorth, J., Sollerman, J., Moller, P., et al. 2003, Nature, 423, 847

Jester, S., Schneider, D. P., Richards, G. T., et al. 2005, AJ, 130, 873

Jin, Z.-P., Covino, S., Della Valle, M., et al. 2013, ApJ, 774, 114

Kawamuro, T., Shidatsu, M., Nakahira, S., et al. 2013, GRB Coordinates

Network, Circular Service, 14462,

Kouveliotou, C. Granot, J. Racusin, J. L., et al. 2013, ApJ, 779, 1

Laskar, T., Berger, E., Zauderer, B. A., et al. 2013, ApJ, 776, 119

Lazzati, D., Covino, S., Ghisellini, G., et al. 2001, A\&A, 378, 996

Levan, A. J., Cenko, S. B., Perley, D. A., \& Tanvir, N. R. 2013a, GRB Coordinates Network, Circular Service, 14455, 1

Levan, A. J., Tanvir, N. R., Fruchter, A. S., et al. 2013b, ApJ, submitted [arXiv: 1307.5338]

Levesque, E. M., Soderberg, A. M., Kewley, L. J., \& Berger, E. 2010, ApJ, 725, 1337

Malesani, D., Tagliaferri, G., Chincarini, G., et al. 2004, ApJ, 609, 5

Maselli, A., Beardmore, A. P., Lien, A. Y., et al. 2013, GRB Coordinates Network, Circular Service, 14448, 1

Maselli, A., Melandri, A., Nava, L., et al. 2014, Science, 343, 48

Masetti, N., Palazzi, E., Pian, E., et al. 2003, A\&A, 404, 465

Matheson, T., Garnavich, P. M., Stanek, K. Z., et al. 2003, ApJ, 599, 394

Mazzali, P. A., Deng, J., Maeda, K., et al. 2002, ApJ, 572, 61

Mazzali, P. A., Deng, J., Tominaga, N., et al. 2003, ApJ, 599, 95

Mazzali, P. A., Deng, J., Pian, E., et al. 2006a, ApJ, 645, 1323

Mazzali, P. A., Deng, J., Nomoto, K., et al. 2006b, Nature, 442, 1018

Mazzali, P. A., Kawabata, K. S., Maeda, K., et al. 2007, ApJ, 670, 592

Mazzali, P. A., Walker, E. S., Pian, E., et al. 2013, MNRAS, 432, 2463

Melandri, A., Pian, E., Ferrero, P., et al. 2012, A\&A, 547, A82

Mirabal, N., Halpern, J. P., An, D., Thorstensen, J. R, \& Terndrup, D. M. 2006, ApJ, 643, 99

Olivares, E. F., Greiner, J., Schady, P., et al. 2011, PIAU, 7, 375

Panaitescu, A., Vestrand, W. T., \& Woźniak, P., 2013, MNRAS, 436, 3106

Patat, F., Cappellaro, E., Danziger, J., et al. 2001, ApJ, 555, 900

Perley, D. A., Cenko, S. B., Corsi, A., et al. 2013, ApJ, 781, 37

Pian, E., Mazzali, P. A., Masetti, N., et al. 2006, Nature, 442, 1011

Pozanenko, A., Minaev, P., Volnova, A., et al. 2013, GRB Coordinates Network, Circular Service, 14484

Preece, R., Burgess, J. M., von Kienlin, A., et al. 2014, Science, 343, 51

Richmond, M. W., van Dyk, S. D., Ho, W., et al. 1996, AJ, 111, 327

Savaglio, S, Glazebrook, K., \& Le Borgne, D. 2009, ApJ, 691, 182

Schlafly, E. F., \& Finkbeiner, D. P. 2011, ApJ, 737, 103

Schlegel, D. J., Finkbeiner, D. P., \& Davis, M. 1998, ApJ, 500, 525

Schulze, S., Malesani, D., Cucchiara, A., et al. 2014, A\&A, 566, A102

Smith, D. M., Csillaghy, A., Hurley, K., et al. 2013, GRB Coordinates Network, Circular Service, 14590

Soderberg, A. M., Kulkarni, S. R., Berger, E., et al. 2004, ApJ, 606, 994

Soderberg, A. M., Kulkarni, S. R., Fox, D. B., et al. 2005, ApJ, 627, 877

Soderberg, A. M., Kulkarni, S. R., Price, P. A., et al. 2006, ApJ, 636, 391

Sollerman, J., Jaunsen, A. O., Fynbo, J. P. U., et al. 2006, A\&A, 454, 503

Sparre, M., Sollerman, J., Fynbo, J. P. U., et al. 2011, ApJ, 735, 24

Stanek, K. Z., Matheson, T., Garnavich, P. M., et al. 2003, ApJ, 591, 17

Starling, R. L. C., Wiersema, K., Levan, A. J., et al. 2011, MNRAS, 411, 2792

Tanvir, N. R., Rol, E., Levan, A. J., et al. 2010, ApJ, 725, 625

Valenti, S., Benetti, S., Cappellaro, E., et al. 2008, MNRAS, 383, 1485

Vanderspek, R., Sakamoto, T., Barraud, C., et al. 2004, ApJ, 617, 1251

Verrecchia, F., Pittori, C., Giuliani, A., et al. 2013, GRB Coordinates Network, Circular Service, 14515

Vestrand, W. T., Wren, J., Panaitescu, A., et al. 2014, Science, 343, 38

von Kienlin, A. 2013, GRB Coordinates Network, Circular Service, 14473

Woosley, S. E., \& Bloom, J. S. 2006, ARA\&A, 44, 507

$\mathrm{Xu}$, D., de Ugarte Postigo, A., Schulze, S., et al. 2013a, GRB Coordinates Network, Circular Service, 14478

Xu, D., de Ugarte Postigo, A., Leloudas, G., et al. 2013b, ApJ, 776, 98

Yonetoku D., Murakami T., Nakamura T., et al. 2004, ApJ, 609, 935

Zeh, A., Klose, S., \& Hartmann, D. H. 2004, ApJ, 609, 952

Zhu, S., Racusin, J., Kocevski, D., et al. 2013, GRB Coordinates Network, Circular Service, 14471 
Table 3. Observation log.

\begin{tabular}{|c|c|c|c|c|c|c|}
\hline Date & $\Delta t$ & $t_{\exp }$ & Magnitude & Flux density & Ref. & Seeing \\
\hline & (days) & $(\min )$ & & $(\mu \mathrm{Jy})$ & & $\left({ }^{\prime \prime}\right)$ \\
\hline 2013 May 3 & 6.722 & 1.0 & $20.93 \pm 0.02$ & $19.4 \pm 0.4$ & VLT- $B$ & 0.96 \\
\hline 2013 Мау 6 & 9.723 & 1.0 & $21.37 \pm 0.04$ & $12.9 \pm 0.5$ & VLT- $B$ & 1.18 \\
\hline 2013 May 8 & 11.645 & 1.0 & $21.40 \pm 0.04$ & $12.5 \pm 0.5$ & VLT- $B$ & 0.77 \\
\hline 2013 May 10 & 13.648 & 1.0 & $21.58 \pm 0.06$ & $10.7 \pm 0.6$ & VLT- $B$ & 1.20 \\
\hline 2013 May 11 & 14.648 & 1.0 & $21.63 \pm 0.12$ & $10.2 \pm 1.1$ & VLT- $B$ & 1.57 \\
\hline 2013 May 13 & 16.644 & 1.5 & $21.74 \pm 0.06$ & $9.3 \pm 0.5$ & VLT- $B$ & 1.49 \\
\hline 2013 May 14 & 17.748 & 1.5 & $21.68 \pm 0.04$ & $9.7 \pm 0.4$ & VLT- $B$ & 0.80 \\
\hline 2013 May 15 & 18.720 & 1.5 & $21.75 \pm 0.08$ & $9.2 \pm 0.7$ & VLT- $B$ & 1.34 \\
\hline 2013 Мay 19 & 22.597 & 2.5 & $21.68 \pm 0.08$ & $9.7 \pm 0.7$ & TNG- $g^{\prime}$ & 1.25 \\
\hline 2013 May 20 & 23.674 & 3.0 & $21.82 \pm 0.22$ & $8.6 \pm 1.8$ & VLT- $B$ & 1.00 \\
\hline 2013 May 25 & 28.657 & 1.5 & $22.11 \pm 0.19$ & $6.6 \pm 1.2$ & VLT- $B$ & 1.11 \\
\hline 2013 May 27 & 30.643 & 1.5 & $22.13 \pm 0.07$ & $6.4 \pm 0.5$ & VLT- $B$ & 1.38 \\
\hline 2013 May 28 & 31.647 & 1.5 & $22.22 \pm 0.04$ & $5.9 \pm 0.2$ & VLT- $B$ & 0.70 \\
\hline 2013 May 29 & 32.637 & 1.5 & $22.26 \pm 0.07$ & $5.7 \pm 0.4$ & VLT- $B$ & 0.96 \\
\hline 2013 June 1 & 34.649 & 1.5 & $22.25 \pm 0.14$ & $5.7 \pm 0.7$ & VLT- $B$ & 0.75 \\
\hline 2013 June 2 & 35.649 & 1.5 & $22.21 \pm 0.06$ & $6.0 \pm 0.3$ & VLT- $B$ & 0.97 \\
\hline 2013 June 3 & 37.649 & 1.5 & $22.20 \pm 0.06$ & $6.0 \pm 0.4$ & VLT- $B$ & 0.76 \\
\hline 2013 June 4 & 38.655 & 1.5 & $22.22 \pm 0.08$ & $5.9 \pm 0.4$ & VLT- $B$ & 0.72 \\
\hline 2013 June 5 & 39.649 & 1.5 & $22.22 \pm 0.09$ & $5.9 \pm 0.5$ & VLT- $B$ & 0.93 \\
\hline 2013 June 6 & 40.639 & 2.5 & $22.19 \pm 0.08$ & $6.1 \pm 0.5$ & VLT- $B$ & 0.81 \\
\hline 2013 June 10 & 44.639 & 1.5 & $22.21 \pm 0.09$ & $6.0 \pm 0.5$ & VLT- $B$ & 0.98 \\
\hline 2013 June 13 & 47.626 & 3.0 & $22.21 \pm 0.23$ & $6.0 \pm 1.3$ & VLT- $B$ & 1.29 \\
\hline 2013 June 15 & 49.649 & 1.5 & $22.24 \pm 0.21$ & $5.8 \pm 1.1$ & VLT- $B$ & 1.57 \\
\hline 2013 June 16 & 50.671 & 1.5 & $22.20 \pm 0.18$ & $6.1 \pm 1.0$ & VLT- $B$ & 1.25 \\
\hline 2013 June 17 & 51.670 & 1.5 & $22.19 \pm 0.22$ & $6.1 \pm 1.2$ & VLT- $B$ & 0.96 \\
\hline 2013 May 3 & 6.724 & 1.0 & $20.31 \pm 0.02$ & $28.7 \pm 0.6$ & VLT- $V$ & 1.16 \\
\hline 2013 May 6 & 9.724 & 1.0 & $20.60 \pm 0.03$ & $22.2 \pm 0.6$ & VLT- $V$ & 1.20 \\
\hline 2013 Мay 8 & 11.647 & 1.0 & $20.74 \pm 0.02$ & $19.4 \pm 0.5$ & VLT- $V$ & 0.85 \\
\hline 2013 May 10 & 13.650 & 1.0 & $20.81 \pm 0.04$ & $18.2 \pm 0.7$ & VLT- $V$ & 1.37 \\
\hline 2013 May 11 & 14.649 & 1.0 & $20.84 \pm 0.03$ & $17.8 \pm 0.4$ & VLT- $V$ & 1.15 \\
\hline 2013 May 13 & 16.646 & 1.5 & $20.90 \pm 0.03$ & $16.8 \pm 0.5$ & VLT- $V$ & 1.30 \\
\hline 2013 May 14 & 17.751 & 1.5 & $20.96 \pm 0.06$ & $15.9 \pm 0.8$ & VLT- $V$ & 0.89 \\
\hline 2013 May 15 & 18.723 & 1.5 & $20.99 \pm 0.04$ & $15.4 \pm 0.6$ & VLT- $V$ & 1.34 \\
\hline 2013 May 20 & 23.677 & 3.0 & $20.85 \pm 0.13$ & $17.7 \pm 2.2$ & VLT- $V$ & 1.17 \\
\hline 2013 May 25 & 28.657 & 1.5 & $21.05 \pm 0.08$ & $14.7 \pm 1.1$ & VLT- $V$ & 1.07 \\
\hline 2013 May 27 & 30.645 & 1.5 & $21.18 \pm 0.05$ & $13.0 \pm 0.5$ & VLT- $V$ & 1.25 \\
\hline 2013 May 28 & 31.649 & 1.5 & $21.25 \pm 0.03$ & $12.2 \pm 0.3$ & VLT- $V$ & 0.68 \\
\hline 2013 May 29 & 32.639 & 1.5 & $21.25 \pm 0.04$ & $12.2 \pm 0.4$ & VLT- $V$ & 1.03 \\
\hline 2013 June 1 & 34.651 & 1.5 & $21.33 \pm 0.06$ & $11.3 \pm 0.7$ & VLT- $V$ & 0.76 \\
\hline 2013 June 2 & 35.652 & 1.5 & $21.34 \pm 0.04$ & $11.2 \pm 0.4$ & VLT- $V$ & 1.33 \\
\hline 2013 June 3 & 37.651 & 1.5 & $21.33 \pm 0.03$ & $11.3 \pm 0.3$ & VLT- $V$ & 0.75 \\
\hline 2013 June 4 & 38.657 & 1.5 & $21.31 \pm 0.03$ & $11.6 \pm 0.4$ & VLT- $V$ & 0.91 \\
\hline 2013 June 5 & 39.652 & 1.5 & $21.44 \pm 0.04$ & $10.3 \pm 0.4$ & VLT- $V$ & 0.80 \\
\hline 2013 June 6 & 40.643 & 2.5 & $21.37 \pm 0.03$ & $10.9 \pm 0.3$ & VLT- $V$ & 0.86 \\
\hline 2013 June 10 & 44.641 & 1.5 & $21.41 \pm 0.04$ & $10.5 \pm 0.4$ & VLT- $V$ & 0.92 \\
\hline 2013 June 13 & 47.628 & 3.0 & $21.48 \pm 0.08$ & $9.9 \pm 0.7$ & VLT- $V$ & 1.62 \\
\hline 2013 June 15 & 49.652 & 1.5 & $21.38 \pm 0.06$ & $10.8 \pm 0.6$ & VLT- $V$ & 1.62 \\
\hline 2013 June 16 & 50.673 & 1.5 & $21.57 \pm 0.08$ & $9.1 \pm 0.7$ & VLT- $V$ & 0.85 \\
\hline 2013 June 17 & 51.660 & 1.5 & $21.41 \pm 0.08$ & $10.5 \pm 0.8$ & VLT- $V$ & 0.87 \\
\hline 2013 April 30 & 3.651 & 1.0 & $19.43 \pm 0.06$ & $54.8 \pm 3.3$ & TNG- $r^{\prime}$ & 1.56 \\
\hline 2013 May 4 & 6.726 & 1.0 & $20.07 \pm 0.05$ & $30.3 \pm 1.4$ & VLT- $R$ & 0.88 \\
\hline 2013 May 7 & 9.727 & 1.0 & $20.35 \pm 0.03$ & $23.4 \pm 0.6$ & VLT- $R$ & 1.30 \\
\hline 2013 May 8 & 11.649 & 1.0 & $20.38 \pm 0.03$ & $22.8 \pm 0.6$ & VLT- $R$ & 1.03 \\
\hline 2013 May 10 & 13.652 & 1.0 & $20.47 \pm 0.04$ & $21.0 \pm 0.8$ & VLT- $R$ & 1.40 \\
\hline 2013 May 11 & 14.657 & 1.0 & $20.52 \pm 0.04$ & $20.0 \pm 0.7$ & VLT- $R$ & 1.21 \\
\hline 2013 May 13 & 16.650 & 1.5 & $20.57 \pm 0.03$ & $19.1 \pm 0.5$ & VLT- $R$ & 1.12 \\
\hline 2013 May 14 & 17.755 & 1.5 & $20.59 \pm 0.04$ & $18.7 \pm 0.6$ & VLT- $R$ & 0.86 \\
\hline 2013 May 15 & 18.726 & 1.5 & $20.50 \pm 0.09$ & $20.4 \pm 1.7$ & VLT- $R$ & 1.34 \\
\hline 2013 Мay 19 & 22.607 & 1.0 & $20.69 \pm 0.16$ & $17.1 \pm 2.7$ & TNG- $r^{\prime}$ & 1.40 \\
\hline 2013 May 20 & 23.680 & 3.0 & $20.41 \pm 0.12$ & $22.2 \pm 2.5$ & VLT- $R$ & 1.17 \\
\hline 2013 May 25 & 28.660 & 1.5 & $20.72 \pm 0.05$ & $16.7 \pm 0.8$ & VLT- $R$ & 0.90 \\
\hline 2013 May 27 & 30.648 & 1.5 & $20.81 \pm 0.04$ & $15.4 \pm 0.6$ & VLT- $R$ & 1.22 \\
\hline
\end{tabular}


A. Melandri et al.: Diversity of GRB energetics vs. SN homogeneity

Table 3. continued.

\begin{tabular}{|c|c|c|c|c|c|c|}
\hline Date & $\Delta t$ & $t_{\exp }$ & Magnitude & Flux density & Ref. & Seeing \\
\hline & (days) & $(\min )$ & & $(\mu \mathrm{Jy})$ & & $\left({ }^{\prime \prime}\right)$ \\
\hline 2013 Мау 28 & 31.654 & 1.5 & $20.88 \pm 0.02$ & $14.4 \pm 0.3$ & VLT- $R$ & 0.77 \\
\hline 2013 May 29 & 32.757 & 1.5 & $20.20 \pm 0.03$ & $14.2 \pm 0.4$ & VLT- $R$ & 0.97 \\
\hline 2013 June 1 & 34.655 & 1.5 & $20.91 \pm 0.06$ & $14.0 \pm 0.7$ & VLT- $R$ & 0.77 \\
\hline 2013 June 2 & 35.656 & 1.5 & $20.92 \pm 0.04$ & $13.9 \pm 0.6$ & VLT- $R$ & 1.18 \\
\hline 2013 June 3 & 37.654 & 1.5 & $20.88 \pm 0.02$ & $14.3 \pm 0.3$ & VLT- $R$ & 0.70 \\
\hline 2013 June 4 & 38.660 & 1.5 & $20.94 \pm 0.03$ & $13.6 \pm 0.4$ & VLT- $R$ & 0.87 \\
\hline 2013 June 5 & 39.651 & 1.5 & $20.94 \pm 0.03$ & & VLT- $R$ & 0.79 \\
\hline 2013 June 6 & 40.642 & 2.5 & $20.95 \pm 0.02$ & $13.5 \pm 0.3$ & VLT- $R$ & 0.89 \\
\hline 2013 June 10 & 44.640 & 1.5 & $21.09 \pm 0.04$ & $11.8 \pm 0.5$ & VLT- $R$ & 1.00 \\
\hline 2013 June 13 & 47.627 & 3.0 & $21.05 \pm 0.06$ & $12.3 \pm 0.7$ & VLT- $R$ & 1.34 \\
\hline 2013 June 15 & 49.651 & 1.5 & $21.16 \pm 0.09$ & $11.1 \pm 0.9$ & VLT- $R$ & 1.56 \\
\hline 2013 June 16 & 50.672 & 1.5 & $21.10 \pm 0.07$ & $11.7 \pm 0.8$ & VLT- $R$ & 1.24 \\
\hline 2013 June 17 & 51.659 & 1.5 & $21.24 \pm 0.05$ & $10.3 \pm 0.5$ & VLT- $R$ & 0.90 \\
\hline 2013 April 30 & 3.652 & 1.0 & $18.95 \pm 0.06$ & $65.7 \pm 3.9$ & TNG- $i^{\prime}$ & 1.34 \\
\hline 2013 May 3 & 6.727 & 1.0 & $19.40 \pm 0.02$ & $43.6 \pm 0.9$ & VLT- $I$ & 0.91 \\
\hline 2013 May 6 & 9.728 & 1.0 & $19.68 \pm 0.03$ & $33.7 \pm 1.0$ & VLT-I & 1.14 \\
\hline 2013 May 8 & 11.650 & 1.0 & $5 \pm 0.03$ & $28.8 \pm 0.8$ & VLT-I & 0.79 \\
\hline 2013 May 10 & 13.653 & 1.0 & $19.85 \pm 0.04$ & $28.7 \pm 1.1$ & VLT-I & 1.40 \\
\hline 2013 May 11 & 14.658 & 1.0 & $19.85 \pm 0.03$ & $28.9 \pm 0.9$ & VLT- $I$ & 0.73 \\
\hline 2013 May 13 & 16.651 & 1.5 & $19.90 \pm 0.05$ & $27.4 \pm 1.3$ & VLT-I & 0.89 \\
\hline 2013 May 14 & 17.756 & 1.5 & $19.88 \pm 0.06$ & $28.0 \pm 1.7$ & VLT-I & 0.94 \\
\hline 2013 May 15 & 18.727 & 1.5 & $20.07 \pm 0.13$ & $24.7 \pm 2.6$ & VLT- $I$ & 1.34 \\
\hline 2013 May 19 & 22.593 & 1.0 & $20.02 \pm 0.16$ & $24.6 \pm 3.6$ & TNG- $i^{\prime}$ & 1.16 \\
\hline 2013 May 20 & 23.681 & 3.0 & $19.83 \pm 0.16$ & $29.3 \pm 4.5$ & VLT-I & 1.17 \\
\hline 2013 May 25 & 28.661 & 1.5 & $19.88 \pm 0.03$ & $28.0 \pm 0.9$ & VLT-I & 0.92 \\
\hline 2013 May 27 & 30.649 & 1.5 & $19.93 \pm 0.04$ & $26.7 \pm 1.1$ & VLT- $I$ & 1.46 \\
\hline 2013 May 28 & 31.655 & 1.5 & $20.07 \pm 0.03$ & $23.4 \pm 0.7$ & VLT-I & 0.86 \\
\hline 2013 May 29 & 32.758 & 1.5 & $20.13 \pm 0.04$ & $22.3 \pm 0.9$ & VLT-I & 1.00 \\
\hline 2013 June 1 & 34.656 & 1.5 & $20.13 \pm 0.05$ & $22.2 \pm 0.9$ & VLT- $I$ & 0.71 \\
\hline 2013 June 2 & 35.657 & 1.5 & $19.96 \pm 0.04$ & $26.0 \pm 1.1$ & VLT- $I$ & 1.25 \\
\hline 2013 June 3 & 37.655 & 1.5 & $20.30 \pm 0.03$ & $19.1 \pm 0.6$ & VLT- $I$ & 0.69 \\
\hline 2013 June 4 & 38.661 & 1.5 & $20.18 \pm 0.04$ & $21.2 \pm 0.8$ & VLT-I & 0.96 \\
\hline 2013 June 5 & 39.652 & 1.5 & $20.27 \pm 0.03$ & $19.5 \pm 0.6$ & VLT-I & 0.76 \\
\hline 2013 June 6 & 40.643 & 2.5 & $20.13 \pm 0$. & $22.2 \pm 0.7$ & VLT-I & 0.91 \\
\hline 2013 June 10 & 44.641 & 1.5 & $20.25 \pm 0.04$ & $19.9 \pm 0.8$ & VLT-I & 0.88 \\
\hline 2013 June 13 & 47.628 & 3.0 & $20.30 \pm 0.03$ & $18.9 \pm 0.5$ & VLT-I & 1.34 \\
\hline 2013 June 15 & 49.652 & 1.5 & $20.33 \pm 0.04$ & $18.5 \pm 0.7$ & VLT- $I$ & 1.49 \\
\hline 2013 June 16 & 50.673 & 1.5 & $20.35 \pm 0.04$ & $18.2 \pm 0.6$ & VLT-I & 0.94 \\
\hline 2013 June 17 & 51.660 & 1.5 & $20.27 \pm 0.03$ & $19.5 \pm 0.6$ & VLT-I & 1.09 \\
\hline
\end{tabular}

